Ophthalmologe 2015 · 112:57-60

DOI 10.1007/s00347-014-3096-y

Online publiziert: 4. Oktober 2014

(c) The Author 2014. This article is published

with open access at Springerlink.com

\author{
K. Kirschfeld \\ Max-Planck-Institut für biologische Kybernetik, Tübingen
}

\title{
Behandlung einer serösen makulären Netzhautablösung mit Antihistaminika
}

vor dem Auftreten des Skotoms der Fall war (• Tab. 1). Die Besonderheit besteht darin, dass der Patient weiß, dass seine Kopfschmerzen durch Histamin ausgelöst werden. Er leidet an einer Histaminintoleranz, die durch eine niedrige Konzentration von Diaminooxidase im Blut bedingt ist (• Tab. 1). Diätfehler, die sich nicht immer vermeiden lassen, führen zu Kopfschmerzen, die vom behandelnden Neurologen als Cluster-Kopfschmerzen klassifiziert werden, ausgelöst durch eine zu hohe Histaminkonzentration im Blutplasma. Durch den Serotoninagonisten Sumatriptan lassen sie sich sicher kupieren.

Wie die Kopfschmerzen in der Nacht vor der Beobachtung des Skotoms anzeigen, war die Histaminkonzentration beim Patienten damals erhöht. Dieses Histamin konnte in die Choriokapillaren gelangen und dort womöglich seine typischen Wirkungen entfalten: Weitung der Kapillaren und Erhöhung ihrer Permeabilität [5] mit Auswirkungen womöglich auch auf das Pigmentepithel. Der Patient vermutete deshalb, dass Histamin ursächlich an der Bildung des serösen Exsudats im Bereich der Fovea beteiligt sein könnte, und ließ den Histamingehalt in Blut und Stuhl bestimmen (• Tab. 1).

Die gefundene Diaminooxidase-Konzentration von $10 \mathrm{U} / \mathrm{ml}$ ist nicht sehr niedrig; bei dieser Konzentration wird trotzdem eine Histaminunverträglichkeit als wahrscheinlich angegeben. Die nüchtern gemessene Histaminkonzentration im Plasma betrug $0,28 \mu \mathrm{g} / \mathrm{l}$, ein Wert, der unter dem mittleren Referenzwert liegt. Die nüchtern gemessene Histaminkonzentration im Stuhl, die man als Mittel- wert über einen längeren Zeitraum interpretieren kann, betrug $555 \mathrm{ng} / \mathrm{g}$. Dieser Wert liegt nahe der Grenze zum pathologischen Befund $(600 \mathrm{ng} / \mathrm{g})$. Wäre die Histaminkonzentration im Blut im Durchschnitt so niedrig gewesen, wie im Nüchternzustand gemessen, so wäre eine niedrigere Histaminkonzentration im Stuhl zu erwarten. Dass sie so hoch lag, deutet darauf hin, dass die Plasmahistaminkonzentration zeitweise erhöht war.

Eine starke Erhöhung der Histaminkonzentration wird als Cluster-Kopfschmerz klinisch manifest und hatte womöglich die CCS ausgelöst. Eine weniger starke Erhöhung, die je nach Diät auftreten kann, braucht nicht zu Kopfschmerzen führen, könnte aber Folgendes bewirken: Ob das seröse Exsudat unter der Rezeptor/Pigmentepithelschicht wieder verschwindet, wird von der Balance zwischen Permeation seröser Blutbestandteile aus den Choriokapillaren bzw. dem Pigmentepithel einerseits und deren Rückresorption andererseits abhängen.

Eine erhöhte Histaminkonzentration könnte dieses Gleichgewicht in Rich-

Der Autor ist der Patient, der seinen Fall hier beschreibt. Die Diagnose wurde von Prof. K.U. Bartz-Schmidt, Universitäts-Augenklinik Tübingen, erstellt. Ich danke ihm für den Hinweis auf einen möglichen Zusammenhang zwischen Histamin und Karboanhydrase IV. Prof. E. Zrenner danke ich für die Diskussion.

\section{Open Access}

This article is distributed under the terms of the Creative Commons Attribution License which permits any use, distribution, and reproduction in any medium, provided the original author(s)

and the source are credited. häufig an Kopfschmerzen leiden [4], was auch bei unserem Patienten in der Nacht 


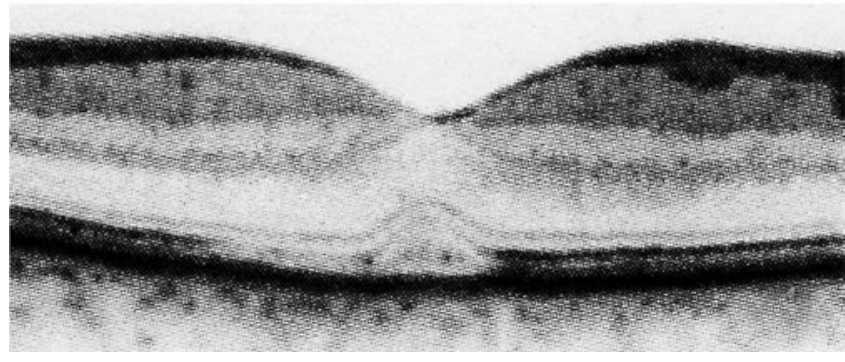

Abb. $1 \Delta$ Optische Kohärenztomographie (OCT) des rechten Auges, vertikal orientiert. Aufnahme vom 24.07.2013: subretinale und retinale seröse Flüssigkeitsansammlungen im fovealen Bereich sind deutlich erkennbar

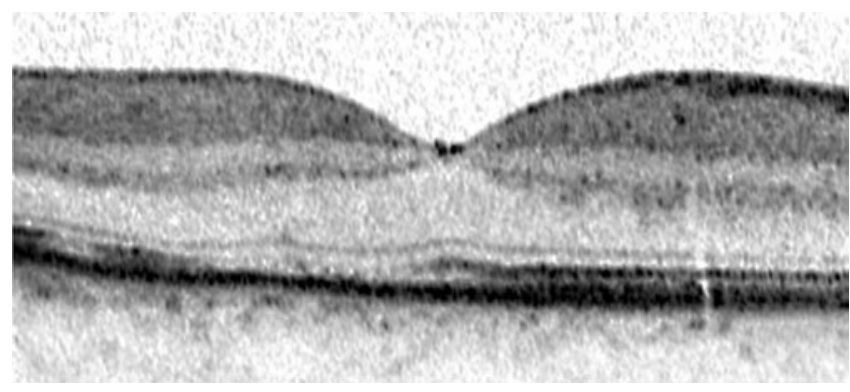

Abb. $3 \Delta$ Optische Kohärenztomographie (OCT) des rechten Auges, horizontal orientiert. Aufnahme vom 08.08.2013: 1 Woche nach der Medikation ist die Flüssigkeitsansammlung fast vollständig abgebaut

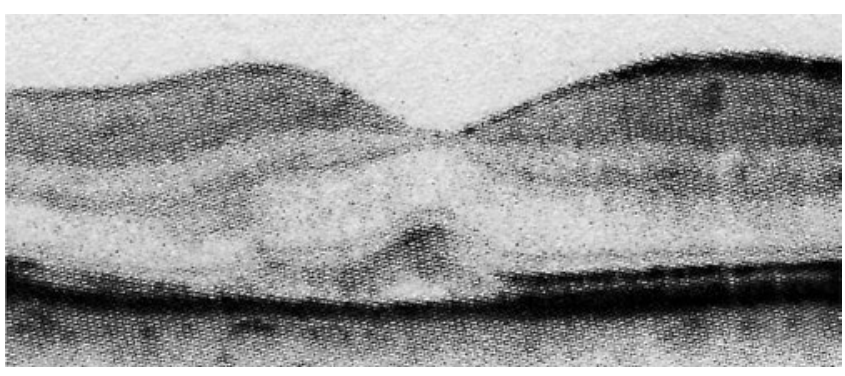

Abb. $2 \Delta$ Optische Kohärenztomographie (OCT) des rechten Auges, horizontal orientiert. Aufnahme vom 24.07.2013: subretinale und retinale seröse Flüssigkeitsansammlungen im fovealen Bereich sind deutlich erkennbar

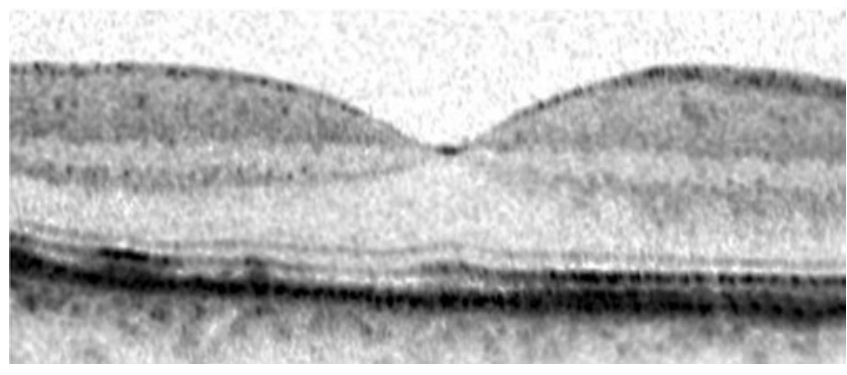

Abb. $4 \Delta$ Optische Kohärenztomographie (OCT) des rechten Auges, horizontal orientiert. Aufnahme vom 23.09.2013: Die Flüssigkeitsansammlung ist kaum mehr erkennbar tung Permeation verschieben, wodurch der Abbau des Exsudats im fovealen Bereich verhindert oder verlangsamt wird. Bei hinreichend hoher Histaminkonzentration könnte auch eine Zunahme des Exsudats auftreten. Gelänge es, das Gleichgewicht zugunsten der Rückresorption zu verändern, so müsste dies den Abbau des Exsudats begünstigen. Wenn Histamin an der Entstehung der CCS beteiligt war, so könnte sich folglich ein Antihistaminikum günstig auf die Rückbildung der CCS auswirken. Der Patient hat deshalb das Antihistaminikum Loratadin eingenommen.

\section{Krankheitsverlauf}

Bei der Erstbeobachtung des Skotoms war es absolut, Lesen mit dem betroffenen Auge war erschwert noch möglich. Im Verlauf von 5 Tagen wurde das Skotom „transparent“, beim Lesen wurde Schrift in ihm erkennbar, wenn auch stark verzerrt. Nach weiteren 5 Wochen hatte sich der betroffene retinale Bereich etwas weiter zur Fovea hin ausgedehnt, Lesen mit diesem Auge war gerade noch möglich. Den Zustand im OCT-Bild zeigen die
- Abb. 1 und 2, seröse Flüssigkeitsansammlungen im Bereich der Fovea sind erkennbar.

In der Zwischenzeit war dem Patienten klar geworden, dass sich ein Antihistaminikum womöglich günstig auswirken könnte. Er nahm deshalb 6 Wochen nach Erstbeobachtung des Skotoms abends $10 \mathrm{mg}$ Loratadin ein. Bereits $5 \mathrm{~h}$ später in der Nacht waren die Metamorphopsien im Amsel-Gitter abgeschwächt, 5 Tage nach täglicher Einnahme des Antihistaminikums (10 mg/Tag, bei 2 vollen Mahlzeiten am Tag gelegentlich 2-mal $10 \mathrm{mg} /$ Tag) waren die serösen Flüssigkeitsansammlungen weitgehend abgebaut, aber noch erkennbar (OCT, • Abb. 3). Lesen mit dem betroffenen Auge war wieder ohne Weiteres möglich, die Bilder von Zeilen beider Augen wurden wieder fusioniert. Der korrigierte Visus betrug dabei auf beiden Augen 1,0. Die OCT vom 23.09.2013 lässt noch eine leichte weitere Verbesserung erkennen (• Abb. 4).

\section{Diskussion}

Seit der Erstbeschreibung der „zentralen rezidivierenden Retinitis“ durch Graefe im Jahr 1866 [6] blieb die Ätiologie dieser Erkrankung unbekannt [1, 2, 9]. Allgemein akzeptiert sind 3 Tatbestände:

1. dass der Abhebung von Rezeptor/Pigmentepithel-Schicht durch Exsudatbildung eine Hyperpermeabilität der Choriokapillaren zugrunde liegt,

2. dass betroffene Patienten häufig an Kopfschmerzen leiden sowie

3. dass die Erkrankung durch psychische Belastung (Stress) ausgelöst werden kann [4].

Unser Patient wusste, dass bei ihm die Cluster-Kopfschmerzen durch Histamin verursacht werden, aber auch, dass Histamin die Permeabilität von Blutkapillaren erhöht [5], sowie dass Stress zu Histaminfreisetzung führt [3]. Alle 3 Tatbestände wären also durch eine erhöhte Histaminkonzentration erklärbar. Dies führte zur Hypothese, dass eine erhöhte Histaminkonzentration im Blutplasma zur Exsudatbildung im fovealen Bereich führen könnte. Daraus ergab sich das Konzept der Antihistaminbehandlung der CCS. Der Erfolg, wie er hier beschrieben und dokumentiert wird, stützt die Histaminhypothese der Genese der CCS. 
Eine zeitliche Nähe ergab sich

- zwischen einem erhöhten Histamingehalt (erschlossen aus den ClusterKopfschmerzen) und dem Auftreten der CCS und

- zwischen der Antihistaminmedikation und einer Verbesserung sowohl der Metamorphopsien (nach Stunden) als auch des OCT-Befundes (nach 1 Woche, $\bullet$ Abb. 3 ).

Auch diese Synchronisationen stützen die Annahme, dass die CCS histaminbedingt sein könnte. Trotzdem sind bei einer solchen Einzelfallbeobachtung Zufälle nicht ausgeschlossen. Weitere Untersuchungen sind deshalb unumgänglich, die gezielt die Möglichkeit einer histaminbedingten Genese der serösen makulären Netzhautablösung thematisieren.

Die Erkrankung wurde zunächst als CCS diagnostiziert, wofür die OCT-Bilder (- Abb. 1, 2) sprechen. Allerdings tritt CCS typischerweise in jüngeren Jahren auf, wenn auch Fälle von CCS in höherem Alter beschrieben wurden. Da die Befunde aber nicht immer klar gegen eine Makuladegeneration abgegrenzt werden können, ist auch im hier beschriebenen Fall eine Makuladegeneration nicht ausgeschlossen. Gegen die Makuladegeneration spricht unter anderem das Fehlen von Drusen zwischen Pigmentepithel und Chorioidea. Es wurde erwogen zu versuchen, die Diagnose durch fluoreszenzangiographische Untersuchungen weiter zu erhärten. Das Ergebnis hätte für den $\mathrm{Pa}$ tienten aber keine Konsequenzen gehabt: Wegen der Nähe des retinalen Defektes zur Fovea war die Anwendung von thermischer Laserkoagulation ausgeschlossen, sodass es auch keinen Sinn gemacht hätte, mit angiographischen Verfahren Quellpunkte von Kontrastmittel für eine Koagulation zu bestimmen. Auf die Anwendung der invasiven Verfahren von Fluoreszenz- und Indozyaninangiographie wurde deshalb verzichtet. Dies bedeutet, dass eine mögliche Rolle von Histamin bei Netzhautablösungen geklärt werden sollte, sowohl wenn sie im Kontext von CCS als auch von AMD auftreten.

Bei CCS ist inzwischen die Gabe des Karboanhydrasehemmers Azetazolamid etabliert [1], als weitere Therapieoption werden Mineralokortikoidrezeptoranta-

Ophthalmologe 2015 · 112:57-60 DOI 10.1007/s00347-014-3096-y

(c) The Author 2014. This article is published with open access at Springerlink.com

\section{K. Kirschfeld \\ Behandlung einer serösen makulären Netzhautablösung mit Antihistaminika}

\section{Zusammenfassung}

Die Ätiologie der Netzhautablösungen bei Chorioretinopathia centralis serosa (CCS) ist unbekannt. Von 3 Tatbeständen wird aber ausgegangen: 1) dass der Abhebung der Rezeptor/Pigmentepithel-Schicht durch ein seröses Exsudat eine Hyperpermeabilität der Choriokapillaren zugrunde liegt; 2) dass betroffene Patienten häufig unter Kopfschmerzen leiden; 3) dass die Erkrankung durch Stress begünstigt wird. Eine erhöhte Histaminkonzentration im Blutplasma könnte zu den genannten Konsequenzen führen. Dies führte zur Hypothese, dass Histamin an der Entstehung der CCS beteiligt sein könnte. Nach Einnahme des Antihistaminikums Loratadin erfolgten innerhalb 1 Woche ein deutli- cher Abbau des retinalen Exsudats sowie die Wiederherstellung des Sehvermögens. Dies stützt die Hypothese, dass Histamin an der Entstehung von Netzhautablösungen beteiligt sein könnte. Weitere Untersuchungen müssen klären, ob diese Hypothese zutrifft und ob Antihistaminika womöglich auch bei Makuladegeneration (AMD) eingesetzt werden können.

\section{Schlüsselwörter}

Chorioretinopathia centralis serosa - Makuladegeneration . Histamin · Antihistaminika .

Mineralokortikoidrezeptorantagonisten

\section{Treatment of serous macular retinal detachment with antihistamines}

\section{Abstract}

The etiology of retinal detachment in central serous retinopathy (CSR) is unknown; however, three facts are generally accepted: (1) the serous exudate which raises the layers of the receptors/pigment epithelium is formed due to hyperpermeability in the choriocapillaries, (2) patients frequently suffer from headaches and (3) stress promotes the incidence of CSR. A high blood plasma histamine concentration can cause the abovementioned symptoms which suggests that histamine might provoke CSR. Within 1 week after administration of the antihistamine loratadin a considerable reduction in the retinal exudate and restoration of vision were observed. This sup- ports the hypothesis that histamine could be involved in the process of retinal detachment. Further investigations and large scale clinical trials should clarify if this hypothesis can be proved or disproved and whether antihistamines can be used for age-related macular degeneration (AMD).

\section{Keywords}

Central serous retinopathy - Macular degeneration · Histamine - Antihistamines . Mineral corticoid receptor antagonists gonisten diskutiert [8]. Die Frage ist, ob Histamin im Kontext mit diesen Therapiekonzepten von Bedeutung sein könnte.

Es konnte gezeigt werden, dass mit Azetazolamid behandelte Patienten eine schnellere Resorption der subretinalen Flüssigkeit sowie eine schnellere Visusrehabilitation aufwiesen als unbehandelte Kontrollgruppen. Allerdings verhinderten Nebenwirkungen eine Dauertherapie [1]. Es ist bekannt, dass die Karboanhydrase IV (CA IV) in den Endothelzellen der Choriokapillaren vorhanden ist (nicht im Pigmentepithel) und gemeinsam mit dem „Na+/bicarbonat Co-transporter 1“ (NBC1) eine Funktionseinheit bildet. Über die Beeinflussung des Pigmentepithels bewirken beide eine Stabilisierung des pH-Wertes [10]. Da Histamin die CA IV aktiviert, könnte ein zu hoher Histaminpegel in den Choriokapillaren das Gleichgewicht bei der CA IV/ NBC1-Funktionseinheit so weit stören, dass das Pigmentepithel seine Aufgabe als Blut-Retina-Schranke nicht mehr genügend erfüllen kann und es zur Permeation seröser Bestandteile ins subretinale/ retinale Gewebe kommt. Die Vorstellung, dass Histamin an der Genese subretinaler Deposite beteiligt ist, fügt sich also widerspruchslos in die Vorstellung der Rolle ein, die CA IV bei der CCS spielt. An- 


\begin{tabular}{|c|c|}
\hline 15./16.06.2013 & Cluster-Kopfschmerzen \\
\hline 16.06.2013 & Erstbeobachtung Skotom \\
\hline 19.06.2013 & Arbeitsdiagnose CCS \\
\hline 23.07.2013 & Diaminooxidase $10 \mathrm{U} / \mathrm{ml}$ \\
\hline 24.07.2013 & $\mathrm{OCT}(\bullet$ Abb. 1, 2) \\
\hline 24.07.2013, 9 Uhr, nüchtern & Histamin Stuhl 555 ng/g St. \\
\hline 25.07.2013, 9 Uhr, nüchtern & Histamin Plasma 0,28 $\mu \mathrm{g} / \mathrm{l}$ \\
\hline $01.08 .2013,21 \mathrm{Uhr}$ & Loratadin $10 \mathrm{mg}$, ab hier täglich 10-20 mg \\
\hline 02.08.2013, $1 \mathrm{Uhr}$ & Verbesserung der Metamorphopsien erkennbar \\
\hline 04.08 .2013 & Metamorphopsien noch schwach vorhanden \\
\hline 08.08 .2013 & OCT (• Abb. 3) \\
\hline 23.09.2013 & OCT (• Abb. 4) \\
\hline
\end{tabular}

tihistaminika könnten Karboanhydrasehemmer bei der Therapie womöglich ersetzen, wobei die Hoffnung besteht, dass dabei keine oder weniger Nebenwirkungen auftreten.

Auch Mineralokortikoidrezeptorantagonisten greifen in das Geschehen im Bereich der Chorioidea ein. Der Mineralokortikoid (MR)-Rezeptor wird in den choroidalen Gefäßen exprimiert und reguliert vermutlich das Kalium/NatriumGleichgewicht. Das natürliche Mineralokortikoid Aldosteron führt zu einer Verdickung der choroidalen Gefäße, was durch Aldosteronantagonisten blockiert werden kann. Daraus resultierte die Idee, dass Mineralokortikoidrezeptorantagonisten bei der Therapie von CCS wirksam sein könnten. Die Anwendung des Antagonisten Epleronon als auch die Blockade von MR-Rezeptoren mit Spironolacton erwiesen sich als wirkungsvoll [8]. Ob womöglich ein funktioneller Zusammenhang auch mit Histamin besteht, kann bisher nicht erkannt werden, erscheint aber bei den zahlreichen Mechanismen, die die Vasokonstriktion und Dilatation kontrollieren, nicht ausgeschlossen.

Bei der Anamnese gibt die Frage, ob der Patient an Seekrankheit leidet, womöglich einen ersten Hinweis auf eine Histaminproblematik, da bekannt ist, dass erhöhte Histaminpegel Seekrankheit begünstigen [7].

\section{Fazit für die Praxis}

- Bei Patienten mit seröser makulärer Netzhautablösung, wie sie hier beschrieben ist, sollte die Möglichkeit der Genese der Erkrankung durch Histamin in Betracht gezogen werden, besonders wenn die Erkrankung mit Kopfschmerzen einhergeht.

- Die Behandlung mit einem Antihistaminikum könnte sich als erfolgreich erweisen, besonders wenn die Histaminwerte erhöht sind und/oder eine niedrige Diaminooxidase-Konzentration vorliegt. Gemessene Plasmahistaminpegel müssen nicht notwendigerweise hoch sein, weil tägliche Schwankungen kaum vollständig erfasst werden können.

\section{Korrespondenzadresse}

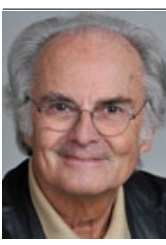

Prof. Dr. K. Kirschfeld

Max-Planck-Institut für biologische Kybernetik Spemannstr. 41, 72076 Tübingen kuno.kirschfeld@ tuebingen.mpg.de

\section{Einhaltung ethischer Richtlinien}

Interessenkonflikt. K. Kirschfeld gibt an, dass kein Interessenkonflikt besteht.

Alle Patienten, die über Bildmaterial oder anderweitige Angaben innerhalb des Manuskripts zu identifizieren sind, haben hierzu ihre schriftliche Einwilligung gegeben. Im Falle von nicht mündigen Patienten liegt die Einwilligung eines Erziehungsberechtigten oder des gesetzlich bestellten Betreuers vor.

Alle im vorliegenden Manuskript beschriebenen Untersuchungen am Menschen wurden mit Zustimmung der zuständigen Ethik-Kommission, im Einklang mit nationalem Recht sowie gemäß der Deklaration von Helsinki von 1975 (in der aktuellen, überarbeiteten Fassung) durchgeführt.

Von allen beteiligten Patienten liegt eine Einverständniserklärung vor.

\section{Literatur}

1. Baraki H, Feltgen N, Roider J et al (2010) Chorioretinopathia centralis serosa (CCS). Ophthalmologe 107:479-493

2. Beger I, Koss MJ, Koch F (2012) Behandlung der Retinopathia centralis serosa. Ophthalmologe 109:1224-1232

3. Black PH, Garbutt LD (2002) Stress, inflammation and cardiovascular disease. J Psychosom Res 52(1):1-23

4. Gass JD (1967) Pathogenesis of disciform detachment of the neuroepithelium. Am J Ophthalmol 63(Suppl):1-139

5. Gotwald T, Coerper S, Schaffer M et al (1998) The mast cell-nerve axis in wound healing: a hypothesis. Wound Repair Regen 6(1):8-20

6. Graefe $A$ (1866) Über die zentrale rezidivierende Retinitis. V Graefes Arch Ophthal 12:211-215

7. Jarisch R (Hrsg) (2013) Histaminintoleranz, Histamin und Seekrankheit. Thieme, Stuttgart

8. Maier M, Stumpfe S, Feucht N et al (2014) Mineralkortikoidrezeptorantagonisten als Therapieoption bei akuter und chronischer Choriaretinopathia centralis serosa. Ophthalmologe 180:173-180

9. Wang M, Munch IC, Hasler PW et al (2008) Central serous chorioretinopathy. Acta Ophthalmol 86:126-145

10. Yang Z, Alvarez BV, Charakova C et al (2005) Mutant carbonic anhydrase 4 impairs $\mathrm{pH}$ regulation and causes retinal photoreceptor degeneration. Hum Mol Genet 14(2):255-265 